\title{
The Optimum Frequency Band Partition Based on Kullback-Leibler Divergence in Subband Image Coding
}

\author{
Haruhiko Miyazaki*, Masashi Kameda \\ Graduate School of Software and Information Science, Iwate Prefectural University \\ *g2311033@s.iwate-pu.ac.jp
}

\begin{abstract}
In order to improve the coding performance of subband image coding, the optimum frequency band partition (OFBP) based on the parameters of subband signal such as signal power or kurtosis has been proposed. The coding performance of two kinds of OFBP is quite different for input image or coding rate. When an input image is given, for instance, OFBP using the signal power is superior at the lower bit rate, on the other hand, OFBP using the kurtosis is superior at the higher bit rate.

In this paper, it is clarified that the similarity between the probability density functions (PDFs) in subband signals is closely related to the improvement of coding performance of the OFBP. We propose a new method to determine the optimum partition pattern on the 2-dimensional frequency domain using the KullbackLeibler divergence (KLD) which is known as a similarity measurement between given two PDFs. It is seen in the experimental results that the PSNR of the proposed method is at most $3.0[\mathrm{~dB}]$ larger than the previous method in OFBP and JPEG at the same coding rate.
\end{abstract}

Keywords: Subband Image Coding, Optimum Frequency Band Partition, Probability Density Function, KullbackLeibler Divergence

\section{Introduction}

Subband coding is one of powerful means for highly efficient coding of image signals[1]. In order to improve the coding performance of the subband coding, it is effective to change the partition pattern on the 2-dimensional frequency domain adaptively in consideration of the stochastic characteristics of input image. Wavelet packet[2] is a representative one in such a method, however, this method has the problem that the computational costs to determine the partition pattern is larger than the general wavelet transform, because the number of subbands in the wavelet packet is too many. In order to the problem of the computational costs in the wavelet packet, the optimum frequency band partition[3] (OFBP) with small number of subbands has been proposed as a method that can determine the optimum partition pattern on the 2-dimensional frequency domain in the view point to maximize the coding gain[4], when the coding rate is given.

Applying the OFBP to any real-world image, first, the 2-dimensional frequency domain is divided into a set of small sized blocks which is called the band blocks. In the next, each subband is reconstructed by the adequate set of band blocks to maximize the coding gain. In our previous method, the signal power or the kurtosis has been used as the parameter for band block signals[5]. It has been seen in the experimental results using the previous method that the partition pattern on the 2-dimensional frequency domain by the signal power is different from the one by the kurtosis. The coding performance of two kinds of OFBP is quite different for input image or coding rate. When an input image is given, for instance, OFBP using the signal power is superior at the lower bit rate, on the other hand, OFBP using the kurtosis is superior at the higher bit rate. The reason why the parameter can't be decided uniquely to obtain the optimum solution that both two parameters are not faithful to the change of the signal in band block, when each subband signal is quantized.

This paper proposes a new OFBP method to minimize the entropy of the coded image, after all band block signals are quantized, namely, the image quality of the coded image is determined. To minimize the entropy in OFBP in our proposed method can solve to minimize the KullbackLeibler divergence[6] (KLD) among the probability density 


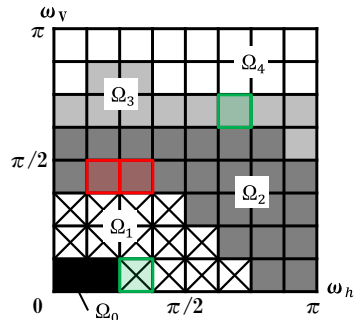

(a) signal power

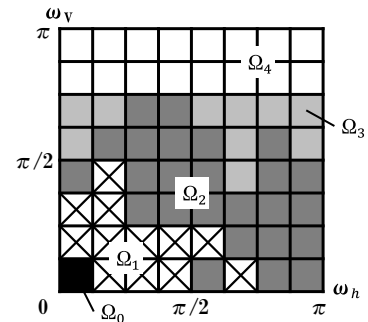

(b) kurtosis
Fig.1. Optimum frequency band partition by previous method (“Lenna").

functions (PDFs) of band block signals in each subband, where KLD is one of similarity measurement between given two PDFs. We propose an algorithm to realize the proposed method which means the optimum partition pattern on the 2-dimensional frequency domain based on the KLD between PDFs of band blocks. In our experiments using some test images, the coding performance by the new partition pattern is improved compared with both the previous method of the OFBP and JPEG.

\section{Optimum Frequency Band Partition}

The optimum frequency band partition[2] (OFBP) is defined as a method that is divided the 2-dimensional frequency domain of an input image into $M$ subbands to maximize the coding gain[4]

$$
G_{V}=10 \log _{10}\left(\frac{\sum_{k=0}^{M-1} \sigma_{k}^{2}}{\prod_{k=0}^{M-1}\left(\sigma_{k}^{2} / \lambda_{k}\right)^{\lambda_{k}}}\right)[d B]
$$

In Eq.(1), $\sigma_{\mathrm{k}}^{2}$ and $\lambda_{\mathrm{k}}$ is the signal power and the coding rate in subband $\Omega_{k}(k=0,1, \ldots, M-1)$, respectively. In order to apply the OFBP to any input image, the optimum solution is realized approximately so that each subband is reconstructed by a set of band blocks $B_{i}(i=0,1, \ldots, N-$ $1 ; N \gg M)$ to maximize the $\mathrm{G}_{\mathrm{V}}$. Here, the band block means a small region on the frequency domain which is calculated by the 2-dimensional separable filter bank using QMF[1]. To maximize the coding gain of Eq.(1) is equivalent with each subband is reconstructed by the set of band blocks with similar signal power.

On the other hand, it is possible to obtain the partition pattern on the 2-dimensional frequency domain using the kurtosis which is an another parameter to express the feature of band block signal instead of the signal power[5]. Fig. 1 shows the examples of the optimum frequency band partition pattern for image "Lenna" in the previous method. Fig.1(a) and Fig.1(b) illustrates the optimum partition pattern based on the signal power and the kurtosis of band blocks, respectively. In this example, band blocks $\mathrm{N}=64$ are classified into subbands $\Omega_{0} \sim \Omega_{4}(\mathrm{M}=5)$, and a linear quantizer with the quantization level=256 is applied to all band blocks. It is seen in Fig. 1 that the partition pattern on the 2-dimensional frequency domain by the signal power is different from one by the kurtosis. Moreover, it has been clarified from the experimental results that the coding performance of two kinds of OFBP is quite different for input image or coding rate. When an input image is given, for instance, OFBP using the signal power is superior at the lower bit rate, on the other hand, OFBP using the kurtosis is superior at the higher bit rate. Therefore, it is necessary to define a new parameter which corresponds to the change of band block signal by the quantization accurately to improve the coding performance in the OFBP.

\section{Proposed Method}

\subsection{Basic Idea}

It is well known that the entropy is given by the probability density function (PDF),

$$
H(X)=-\sum_{x} P(x) \log _{2} P(x)
$$

is used as a measurement amount of information, where $P(x)$ is probability for an event $x$. In order to discuss the entropy, namely, the coding rate in the OFBP, we focus on the PDF of band block signal. When two band blocks whose PDFs are given as $P(x)$ and $Q(x)$, the simple problem in the OFBP is which measure is better

(i) $P(x)$ and $Q(x)$ are grouped into same subband, or

(ii) $P(x)$ and $Q(x)$ are divided into different subbands, in the viewpoint to reduce the total entropy. According to Eq.(2), the entropy in the case of the measure (i) and (ii) is calculated by

$$
H(X)^{(i)}=-\sum_{x} \frac{P(x)+Q(x)}{2} \log _{2}\left[\frac{P(x)+Q(x)}{2}\right]
$$

and

$$
H(X)^{(i i)}=-\sum_{x}\left(\frac{P(x) \log _{2} P(x)+Q(x) \log _{2} Q(x)}{2}\right)
$$

, respectively. If $H(X)^{(i i)}$ is close to $H(X)^{(i)}$, it is desirable to choose the measure (i), because the computational costs by applying band partitioning to an original signal is laborious, in spite of the reduced entropy by the band partitioning is a trifling amount. Contrary to 


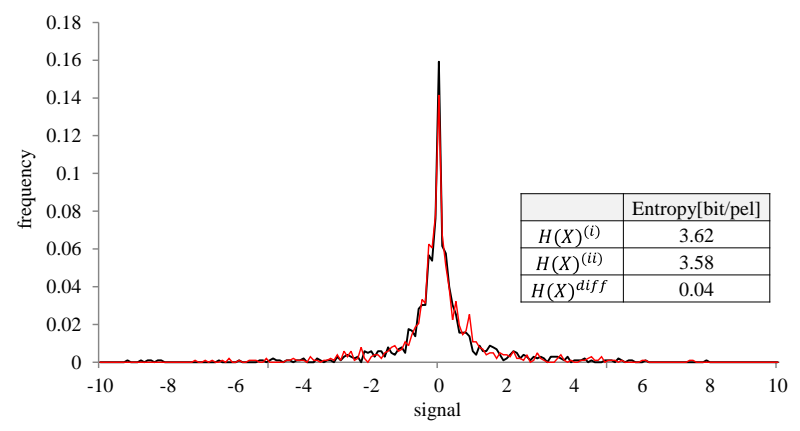

(a) Comparison of two PDFs of red frames in Fig.1.

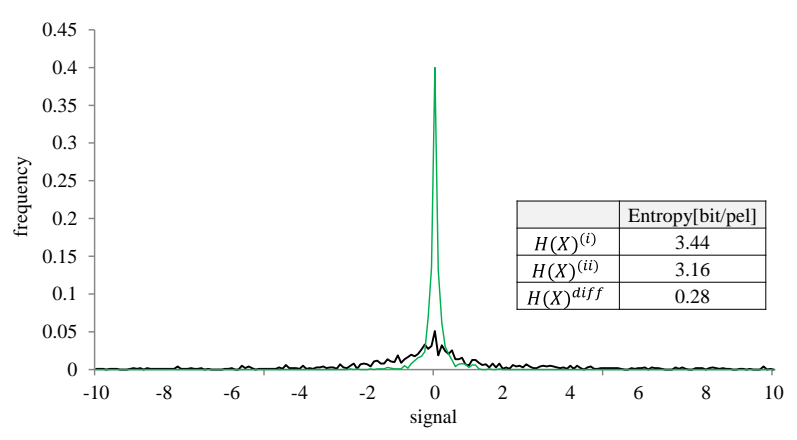

(b) Comparison of two PDFs of green frames in Fig.1.

Fig.2. Comparison of a pair of the probability density functions (PDFs) of band blocks ("Lenna").

this, if $H(X)^{(i i)}$ is much smaller than $H(X)^{(i)}$, it is desirable to choose the measure (ii). In fact, we will show two typical examples by using band blocks for image "Lenna". Fig.2 shows a pair of PDFs of band blocks, where Fig.2(a) and Fig.2(b) is correspond to red frames and green frames are shown in Fig.1, respectively. Moreover, $H(X)^{(i)}$ and $H(X)^{(i i)}$ in Fig.2 denote entropies in the case of Eq.(3) and Eq.(4). And, $H(X)^{\text {diff }}$ is the difference value between $H(X)^{(i)}$ and $H(X)^{(i i)}$, it means the amount of reduced entropy by applying band partitioning. It is seen in Fig.2 that $H(X)^{\text {diff } f}$ in the case of Fig.2(a) is smaller than $H(X)^{\text {diff }}$ in the case of Fig.2(b), and the two PDFs in Fig.2(a) are similar each other. On the other hand, two PDFs in Fig.2(b) are significantly different each other. According this observation, if two PDFs are much different each other, it should choose the measure (ii) to reduce the entropy in the OFBP. Therefore, it is need to define a new parameter which can evaluate the similarity between the PDFs to obtain the optimum frequency band partition in the viewpoint of the entropy minimization. In [6], it is possible to measure the similarity between the PDFs by using the Kullback-Leibler divergence (KLD), which is known as a similarity measurement between given two PDFs. The KLD between two PDFs $P(x)$ and $Q(x)$ is defined by

$$
D_{K L}(P \| Q)=\sum_{x} P(x) \log \frac{P(x)}{Q(x)} .
$$

After the PDFs of all band blocks are given, the OFBP to minimize the total entropy is formulated as the problem to obtain the partition pattern on the 2-dimensional frequency domain to minimize KLD among PDFs of band blocks in each subband.

\subsection{Derivation of New OFBP}

We will formulate the entropy minimization problem in the OFBP under the condition that a same quantizer is applied to all band blocks. Let us define the probability density functions (PDFs) of band blocks $B_{i}(i=0,1, \ldots$, $N-1)$ and subband $\Omega_{k}(k=0,1, \ldots, M-1)$ is as $B_{i}(x)$ and $\Omega_{k}(x)$, respectively. The total entropy for the all subband signals which is obtained by the partition pattern on the 2-dimensional frequency domain can compute by

$$
J_{e}=-\frac{1}{N} \sum_{k=0}^{M-1} R_{k} \sum_{x=\min }^{\max } \Omega_{k}(x) \log _{2} \Omega_{k}(x) .
$$

In Eq.(6), $R_{k}$ is the number of band blocks in subband $\Omega_{k}$, and $\min$ and $\max$ are the minimum and maximum value of $x$ with $\Omega_{k}(x) \neq 0$. Then, PDF of subband $\Omega_{k}(x)$ is replaced by

$$
\Omega_{k}(x)=\frac{1}{R_{k}} \sum_{B_{i} \in \Omega_{k}} B_{i}(x)
$$

using $B_{i}(x)$. Furthermore, when

$$
-p_{m} \log q_{m}=p_{m} \log \frac{p_{m}}{q_{m}}-p_{m} \log p_{m}
$$

is applied to Eq.(6),

$$
\begin{aligned}
J_{e}= & -\frac{1}{N} \sum_{k=0}^{M-1} \sum_{B_{i} \in \Omega_{k}} \sum_{x=\min }^{\max } B_{i}(x) \log _{2} \Omega_{k}(x) \\
= & \frac{1}{N} \sum_{k=0}^{M-1} \sum_{B_{i} \in \Omega_{k}} \sum_{x=\min }^{\max } B_{i}(x) \log _{2} \frac{B_{i}(x)}{\Omega_{k}(x)} \\
& +\left(-\frac{1}{N} \sum_{i=0}^{N} \sum_{x=\text { min }}^{\max } B_{i}(x) \log _{2} B_{i}(x)\right)
\end{aligned}
$$

is obtained. Based on Eq.(5), term 1 in Eq.(9) is considered as the average of Kullback-Leibler divergences (KLDs) between the PDF of each band block $B_{i}(x)$ in $\Omega_{k}(x)$ and PDF of subband $\Omega_{k}(x)$. Also, term 2 in Eq.(9) is the average of entropies using PDFs of all band blocks $B_{i}(x)$. Because term 2 is independent on the result of the partition pattern, minimizing $J_{e}$ of Eq.(9) can be seen as equivalent 
Table.1. PSNR vs. entropy (“Lenna”).

\begin{tabular}{c|c|c|c|c}
\hline \multirow{2}{*}{ Level } & \multicolumn{3}{|c|}{ Entropy[bit/pel] } & \multirow{2}{*}{ PSNR[dB] } \\
\cline { 2 - 4 } & Signal Power & Kurtosis & Proposed Method & \\
\hline \hline 16 & 0.0889 & 0.0897 & $\mathbf{0 . 0 8 8 8}$ & 23.32 \\
\hline 32 & 0.2131 & 0.1880 & $\mathbf{0 . 1 8 6 0}$ & 26.55 \\
\hline 64 & 0.4087 & 0.3995 & $\mathbf{0 . 3 8 4 0}$ & 29.88 \\
\hline 128 & 0.7530 & 0.7398 & $\mathbf{0 . 7 2 4 0}$ & 33.24 \\
\hline 256 & 1.2521 & 1.2492 & $\mathbf{1 . 2 2 6 1}$ & 36.19 \\
\hline
\end{tabular}

Table.2. PSNR vs. entropy ("Barbara").

\begin{tabular}{c|c|c|c|c}
\hline \multirow{2}{*}{ Level } & \multicolumn{3}{|c|}{ Entropy[bit/pel] } & \multirow{2}{*}{ PSNR[dB] } \\
\cline { 2 - 4 } & Signal Power & Kurtosis & Proposed Method & \\
\hline \hline 16 & 0.1029 & 0.1031 & $\mathbf{0 . 1 0 2 8}$ & 22.03 \\
\hline 32 & 0.2458 & 0.2466 & $\mathbf{0 . 2 4 4 9}$ & 25.21 \\
\hline 64 & 0.5101 & 0.5129 & $\mathbf{0 . 5 0 8 8}$ & 28.61 \\
\hline 128 & 0.9381 & 0.9470 & $\mathbf{0 . 9 2 6 3}$ & 31.92 \\
\hline 256 & 1.5545 & 1.6094 & $\mathbf{1 . 5 4 6 4}$ & 34.68 \\
\hline
\end{tabular}

to minimize

$$
G_{K L}=\sum_{k=0}^{M-1} \sum_{B_{i} \in \Omega_{k}} D_{K L}\left(B_{i} \| \Omega_{k}\right) .
$$

To minimize $G_{K L}$ means that each subband $\Omega_{k}$ is reconstructed by the band blocks $B_{i}$ whose PDF are similar each other. Therefore, it is clarified that the entropy of the OFBP can be reduced by minimizing the KLDs among the PDFs of band blocks in each subband.

\subsection{Algorithm}

We propose an algorithm to determine the optimum frequency band partition (OFBP) based on the similarity between the probability density functions (PDFs) of band blocks. However, it is difficult to search the partition pattern to minimize $G_{K L}$ in the viewpoint of the computational costs, because the number of the partition patterns is enormous under the condition of OFBP is $\mathrm{N}=64$ and $M=5$. On the other hand, to maximize the coding gain in Eq.(1) is equivalent that each subband is reconstructed by the set of band blocks with similar value of parameter. In order to maximize the coding gain in Eq.(1) after the signal power of band block is given, first, we have sorted all band blocks in descending order as $\mathrm{S}_{k}=\left\{\Delta s_{0}, \Delta s_{1}, \ldots, \Delta s_{j}, \ldots\right.$, $\left.\Delta s_{N-1}\right\}$, where $\Delta s_{j}$ means the $j$-th band block. In such case, the optimum solution is contained in the restricted partition pattern

$$
\begin{gathered}
S_{k}=[\underbrace{\left\{s_{0}, \Delta s_{1}, \ldots\right\}}_{\left(S_{0, k}\right)}, \ldots, \underbrace{\left\{\ldots, \Delta s_{j}, \ldots\right\}}_{\left(S_{m, k}\right)}, \ldots, \\
\ldots \underbrace{\left\{\ldots, \Delta s_{N-1}\right\}}_{\left(S_{M-1, k}\right)}
\end{gathered}
$$

, where $\left(S_{m, k}\right)$ is the $m$-th subband in the partition pattern $\mathrm{S}_{k}$. Therefore, it is possible to determine the optimum partition pattern with small computational costs by searching the restricted partition pattern under the especial condition of Eq.(11). At this time, the costs required for search can be reduced from $5.42 \times 10^{44}$ to 595,665 under the above condition. Similarly, we determine the optimum partition pattern to minimize $G_{K L}$ in Eq.(10) by sorting the band blocks according to the similarity of PDF. In our proposed method, first, all band blocks is sorted in descending order of signal power, because the signal power is considered as one of parameter which is correspond to the shape of PDF approximately.

\section{Experimental Results}

The proposed method is applied to test image "Lenna" and "Barbara" under the condition that band blocks $N=64$ classified into $\mathrm{M}=5$ subbands, and a linear quantizer with the quantization level $(256,128,64,32,16)$ is applied to all band blocks. Table. 1 and Table. 2 shows the results of the PSNR vs. entropy of the proposed method for "Lenna" and "Barbara", respectively, compared with the previous 


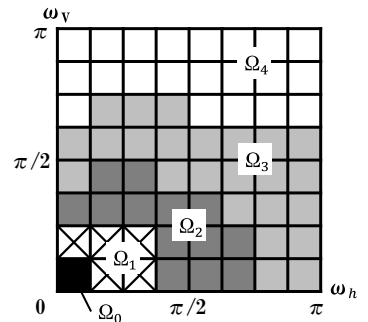

Fig.3. Optimum frequency band partition by proposed method ("Lenna").

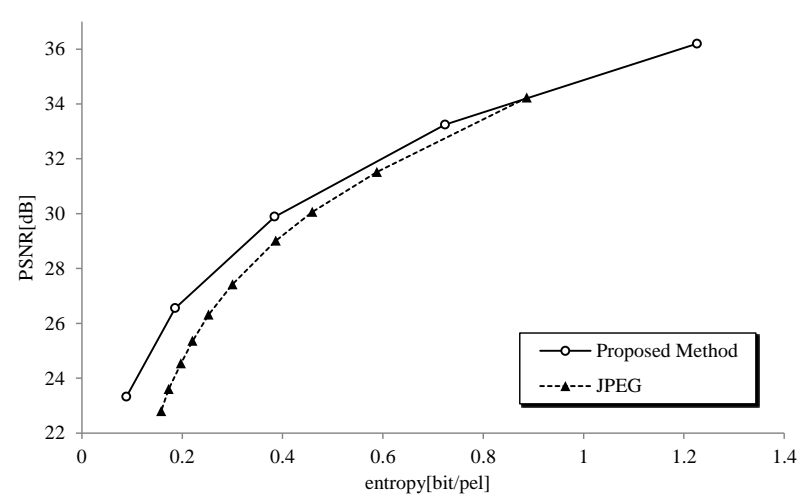

Fig.4. Comparison of coding performance with JPEG ("Lenna").

OFBPs[5] using signal power and kurtosis as parameter of band blocks. It is seen in Table. 1 and Table. 2 that the entropy of the proposed method is reduced than the previous OFBPs.

Fig.3 illustrates the partition pattern of the proposed method for image "Lenna" when a linear quantizer with the quantization level=256 is applied to all band blocks. It is seen in Fig. 3 compared with Fig. 1 that the area of subband $\Omega_{1}$ and $\Omega_{2}$ in Fig.3 tend to be smaller than two partition patterns are shown in Fig.1, because the shape of band block PDFs in the lower-frequency domain are different each other.

Also, Fig.4 shows the PSNR vs. entropy characteristics of the proposed method for "Lenna" compared with JPEG. It is seen in Fig. 4 that the proposed method is superior about 3.0[dB] than JPEG at the lower coding rates.

\section{Conclusion}

In this paper, we have proposed a new optimum frequency band partition (OFBP) method that the partition pattern on the 2-dimensional frequency domain to minimize the entropy for the coded image. It is clarified that the entropy minimization problem in the OFBP can solve to minimize the Kullback-Leibler divergence (KLD) among the probability density functions (PDFs) of band block signals in each subband. In the experimental results, the entropy of the proposed method is reduced than the previous OFBP, and the PSNR of the proposed method is about 3.0[dB] larger than JPEG at the same coding rate.

However, our proposed method OFBP has the problem that the computational costs to determine the partition pattern is larger than the previous OFBP, because the calculation of KLD between PDFs requires a several hundred histogram bins. To propose a new simple parameter which can evaluate the similarity between the PDFs instead of the KLD will be defined as a future work.

\section{References}

[1] J.W.Woods, S.D.O'Neil, "Subband coding of images," IEEE Trans. Accoust., Speech, Signal Processing, vol.ASSP-34, no.5 pp.1278-1288 (1986).

[2] K.Ramchandran, M.Vetterli, "Best wavelet packet basis in a rate-distortion sense," IEEE Trans. Image Process., vol.2, no.2, pp.160-175 (1993).

[3] M.Kameda, K.Ohtake, M.Miyahara, "2-dimensional optimum frequency band partition based on band blocks for subband image coding," IEICE Trans., vol.J81-D-II, no.6, pp.1095-1107 (1998).

[4] N.S.Jayant, P.Noll, "Digital Coding of Waveforms," Prentice Hall, pp.486-509 (1984).

[5] M.Kameda, M.Shirakawa, "Optimum frequency band partition based on kurtosis in subband image coding," IIEEJ Trans., vol.43, no.1, pp.3-12 (2014).

[6] M.N.Do, M.Vetteli, "Wavelet-based texture retrieval using generalized Gaussian density and kullback-leibler distance," IEEE Trans. Image Process., vol.11, no.2, pp.146-158 (2002). 